
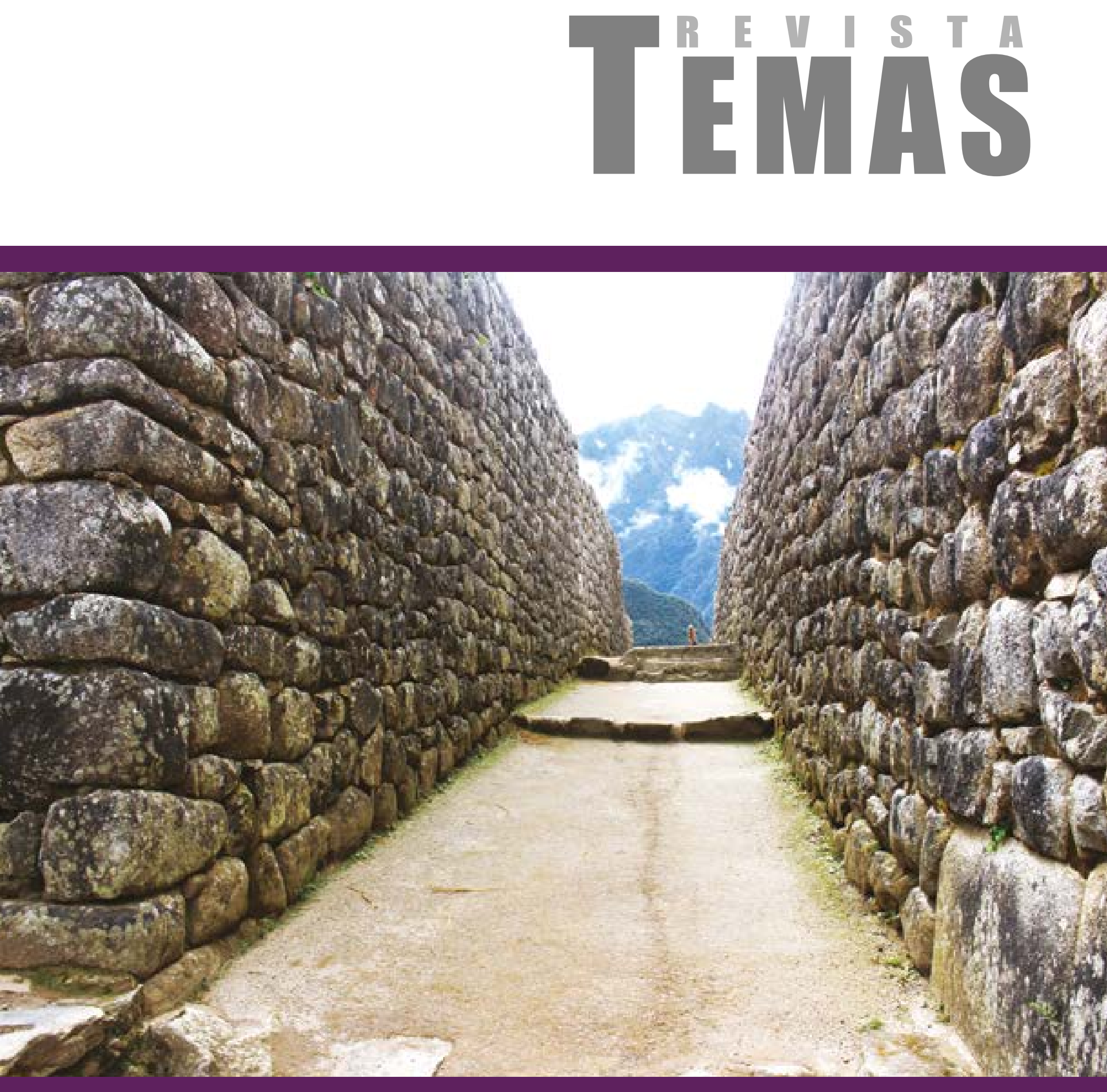

Referencia al citar este artículo:

Velásquez, J., Tarazona, N. (2016). Percepciones y actitudes sobre ética empresarial en los procesos de servicio al cliente liderados por los egresados de la Maestría en Administración de la Universidad Santo Tomás Seccional Bucaramanga. Revista TEMAS, 3(11), 239 - 248

http://dx.doi.org/10.15332/rt.v0i11.1758

\section{Percepciones y actitudes sobre ética empresarial en los procesos de servicio al cliente liderados por los egresados de la Maestría en Administración de la Universidad Santo Tomás Seccional Bucaramanga ${ }^{1}$}

Jhon Jairo Velásquez Ariza ${ }^{2}$ Miguel Ángel Tarazona Méndez ${ }^{3}$

Recibido 31/05/2017 Aprobado 13/07/2017

\section{Resumen}

La reflexión ética del estudio parte del cuestionamiento sobre la responsabilidad de los profesionales que fomentan prácticas indebidas en sectores públicos y privados, y que atentan contra los derechos humanos y el medio ambiente. En ese sentido, mediante un trabajo cuantitativo con alcance exploratorio, y a través de un cuestionario se interrogaron a los egresados de la Maestría en Administración de la Universidad Santo Tomás Seccional Bucaramanga, graduados en los años 2012 y 2013, para conocer cuáles son sus actitudes y percepciones sobre ética empresarial, como líderes de procesos de servicio al cliente en sus empresas.

\section{Palabras clave}

Ética, valores, empresa, responsabilidad, percepción, actitud.

\section{Perceptions and attitudes about business ethics in the processes of customer service led by the graduates of the Masters in Administration of the Santo Tomás University}

\section{Abstract}

The ethical reflection of the study based on the questioning on the responsibility of professionals who encourage improper practices in public and private sectors that threaten human rights and the environment. In this regard through a quantitative work, with exploratory scope and through a questionnaire they surveyed the graduates of the Master in Administration from the Universidad Santo Tomás Bucaramanga branch, graduates in the years 2012 and 2013 to know what their attitudes and perceptions of business ethics as leaders of service processes to the customer in their companies. Thus it was concluded that only in $53 \%$ of the answers of graduates considered that in their organizations care and foster values such as respect, solidarity, justice, responsibility and honesty.

\section{Kevwords}

Ethics, values, enterprise, responsibility, perception, attitude.

1. Artículo de investigación.

2. Magíster en Administración MBA Correo electrónico: j velasquez@outlook.com

3. Magíster en Administración MBA Correo electrónico: mtarazona2009@gmail.com 


\section{Introducción}

El comportamiento ético de los profesionales en su administración y participación en las organizaciones ha sido tema de análisis y de cuestionamiento en distintas esferas de la sociedad a lo largo de la historia. Las noticias de continuos escándalos de corrupción en sus diferentes modalidades como el fraude en los contratos (carrusel de contratistas), licitaciones tramposas, especulación en los precios, lavado de activos, sobrefacturación en compra y venta de productos, incumplimiento de los acuerdos iniciales, evasión tributaria, competencia desleal, apropiación y robo de recursos, salarios injustos, el soborno, falsedad documental, asociación ilícita de uniones temporales, matoneo empresarial, el chantaje, destrucción del medio ambiente y violación de los derechos humanos, develan algunas de las dificultades a las que se enfrentan de manera permanente una gran cantidad de organizaciones públicas y privadas en Colombia, que ponen en riesgo la estabilidad de sus procesos y hasta la calidad de vida y el desarrollo social de su población.

Según lo expuesto por el Índice de Percepción de Corrupción (Transparencia Internacional, 2015) Colombia ocupa el puesto 83 en el índice de corrupción entre 167 países, lugar que lo cataloga como nación con altos índices de corrupción, ya que la calificación obtenida es de 37 , teniendo en cuenta una escala de medición de cero (0) a cien (100), siendo cero la calificación de una percepción de total corrupción y cien una percepción de no corrupción. Esto permite inferir que Colombia en su tejido social a nivel gubernamental y privado carece de credibilidad en la gestión de la transparencia de dichos procesos.
No obstante, se afirma que las empresas que incluyen un modelo ético de responsabilidad social dentro de sus procesos generan beneficios y más ventajas competitivas sobre aquellas que no lo asumen. De esta manera, las que siguen los principios de la responsabilidad social empresarial hacen una caracterización de su cultura organizacional, generan valor, mejoran los procesos internos, reducen costo de gestión y aumentan su reputación al lograr más credibilidad y confianza en sus clientes (Arbaiza, 2010)

Incluso, los empleados son importantes para el buen nombre de la compañía porque invierten en ella tiempo, pasión, entusiasmo, devoción y lealtad. En la mayoría de los casos, pasan la vida entera con ellas (CEPAL, 2008). Por otra parte, su opinión contribuye significativamente a la reputación de la empresa.

Problemas como los enunciados, que son constantes en el país, cuestionan la conducta ética de los individuos y su formación como profesionales; así lo evidencian los continuos escándalos de corrupción que son noticia en todos los medios de comunicación. Es una situación que cuestiona el papel de las instituciones de educación superior en la formación integral que le brinda a sus estudiantes y que debería ser objeto de estudio en algunos escenarios académicos como seminarios, foros, congresos y talleres de formación docente que ofrecen las universidades.

Al respecto, uno de los grandes propósitos del Consejo Nacional de Educación Superior - CESU, consignados en el documento Acuerdo por lo Superior 2034. "Hacia un sistema de educación superior pertinente y de calidad para todos" (2013), en el apartado 6 de la visión, hace especial énfasis en la urgente necesidad de constituir verdaderas comunidades universi- 
tarias que aporten a la cohesión social del país y, por ende, a las instituciones de educación superior y a sus miembros en lo que corresponde al bienestar, permeando todos los procesos para que contribuyan realmente a la formación integral, con privilegio en la formación ética y en los valores expresados en actuaciones de respeto, solidaridad y reconocimiento del otro como interlocutor válido para la reconstrucción de una sociedad justa e incluyente.

Así, lo primero que se debe considerar, es incluir en los planes de estudio de todos los programas académicos asignaturas que promuevan la ética para profesionales y el desarrollo de valores en la experiencia de vida universitaria. Pero esta alternativa es compleja, porque requiere del consenso de las universidades, a través de una política nacional en aras del desarrollo de lo humano.

De igual manera, en el Acuerdo por lo Superior 2034 del CESU se insiste en que las instituciones educativas deben aportar a la formación de personas con visión ética, con sentido de lo público y con capacidad para la convivencia, a fin de generar verdaderas comunidades; un ethos universitario que contribuya al bienestar social de quienes lo integran, con expresiones que surjan y propendan por experiencias de civismo, autonomía, respeto, creatividad y sensibilidad, es decir, de humanidad.

Las instituciones de educación superior no deben escatimar esfuerzos en la formación de sus estudiantes. Se debe buscar que sean profesionales capaces de generar el desarrollo, crecimiento y sostenibilidad de las organizaciones, no solo por medio de su conocimiento y experiencia, también, por su comportamiento y compromiso ético.
En ese sentido, Edgar Morín, sociólogo francés, refiriéndose a la formación indica que el objetivo de las instituciones sociales es formar con base en competencias para afrontar los grandes retos de la humanidad, tales como la cooperación, la solidaridad, el equilibrio ecológico, la disminución de la contaminación, la generación de una cultura de convivencia, la búsqueda de la justicia social y la integración comunitaria en su diversidad (Morín, 2002).

En ese sentido, desde los propósitos de formación, para el caso de la Universidad Santo Tomás y, específicamente, en el diseño curricular del plan de estudio de la Maestría en Administración, los estudiantes reciben orientación y entrenamiento para un ejercicio ético en las organizaciones, a través de asignaturas teóricas y experienciales como Ética Profesional, Ética de los Negocios, Ética Empresarial, Responsabilidad Social, entre otras. De esta manera se llega a pensar que, al tener acceso a los diferentes planes sobre ética, bastará para garantizar un desempeño profesional idóneo.

Pero, ¿cómo aproximarse a esa conceptualización y comprensión de una madurez ética que se expresa en las prácticas profesionales de los egresados de Maestría en Administración de la Universidad Santo Tomás? y ¿qué incidencia tienen estas prácticas en el desarrollo de las organizaciones?

La Universidad Santo Tomás Seccional Bucaramanga promueve entre los estudiantes el desarrollo humano integral, mediante la generación de herramientas investigativas y pedagógicas que, desde el diálogo y la reflexión sobre las realidades, impulsan un pensamiento social fundado en actitudes éticas que aportan a la construcción de la democracia (Universidad Santo Tomás, 2004). 
De esta manera, a través del Departamento de Humanidad es, la Universidad Santo Tomás Seccional Bucaramanga se sitúa en la oferta de diferentes cátedras, a partir de cuatro escenarios estratégicos: Saberes disciplinares especializados, Construcción de racionalidad, Desarrollo humano integral y Ethos vital; escenarios que se fundamentan en cuatro categorías de pensamiento: Complejidad, Decolonialidad, Transdisciplinariedad y Biocentrismo. Con ello se busca alcanzar las competencias específicas de la formación integral desde lo académico, entendidas como un saber hacer en contexto o hábitos operativos: comprender, hacer, obrar y comunicar (USTA, 2014), a fin de dar respuesta al desafío de la formación ética y su compresión antropológica.

Por ejemplo, una cátedra es la de Responsabilidad Social Empresarial que se dicta en la Maestría en Administración, que constituye el insumo institucional en la dimensión ética y pretende brindar las herramientas necesarias para el alcance de estas competencias; pero, a pesar de este gran avance, aún falta establecer un canal de verificación sobre las experiencias y prácticas de los egresados en escenarios reales como son las empresas donde trabajan y la labor en procesos que ponen a prueba su actitud frente al cliente con respecto a los valores de respeto, solidaridad, justicia, responsabilidad y honestidad.

\section{Metodología}

Esta investigación tiene un enfoque cuantitativo con alcance exploratorio y la técnica de recolección de información es la encuesta. El análisis de resultados es cualitativo y se examinan de igual manera las variables sociodemográficas de los egresados encuestados, y desde una perspectiva interpretativa se describe una realidad que ellos manifiestan a través de los cuestionarios aplicados. Además, se determina una variedad de concepciones o marcos de interpretación que hacen parte de un patrón cultural descrito en los objetivos de la investigación.

Además, el estudio es transversal, porque se ha recolectado en un solo momento a través del cuestionario. Este enfoque es adecuado para analizar percepciones. Para la caracterización de los conceptos también se comparan algunos datos etnográficos, como el género, con la prueba estadística t-Student, y mediante la técnica general del análisis de varianza (ANOVA) se analizaron las variaciones de cada una de las variables con respuesta continua de los valores: como: respeto, justicia, solidaridad, responsabilidad y honestidad. Adicional, se considera el diseño de la investigación como no experimental y transaccional exploratorio.

Este tipo de estudios se utilizan justamente para "conocer una variable o un conjunto de variables, una comunidad, un contexto, un evento, una situación, y se trata de una exploración inicial en un momento específico. Por lo general, se aplican a problemas de investigación nuevos o poco conocidos, además, constituyen el preámbulo de otros diseños no experimentales y experimentales" (Sampieri, Fernández, \& Baptista, 2010, p. 152).

La técnica utilizada es la encuesta y el instrumento un cuestionario evaluado mediante el coeficiente Alfa de Cronbach, con el objetivo de medir su confiabilidad, debido a que ha sido adaptado para el proyecto de investigación de acuerdo con su contexto al momento de aplicarlo a los egresados de la Maestría en Administración para identificar las percepciones y actitudes sobre los conceptos enunciados por el filósofo Horacio Martínez Herrera, respecto al paradigma de los valores para la ética, como: respeto, justicia, solidaridad, responsabilidad y honestidad. 
El perfil de la población corresponde a: egresados de la Maestría en Administración de la Universidad Santo Tomás Seccional Bucaramanga, graduados en los años 2012 y 2013, que lideran procesos de servicio al cliente en empresas ubicadas en el área metropolitana de Bucaramanga. De acuerdo con lo anterior, la población estuvo constituida por 12 egresados que cumplían con el perfil. El tipo de muestreo fue no probabilístico, determinado por un censo que se realizó a través de llamadas telefónicas. Como resultado de este censo se tuvo acceso a 9 sujetos que corresponden al $75 \%$ de la población y que constituyeron la muestra de esta investigación. La muestra es homogénea, esto significa que las unidades por seleccionar poseen un mismo perfil o características, o bien, comparten rasgos similares.

\section{Resultados}

A través de este estudio se analizan las percepciones de algunos egresados de la Maestría en Administración sobre ética empresarial, en los procesos que lideran y permiten evidenciar sus actitudes con respecto al servicio que ofrecen a los clientes en sus organizaciones, teniendo en cuenta en esa relación los valores de respeto, solidaridad, justicia, responsabilidad y honestidad.

Desde este punto de vista se han revisado las respuestas de los egresados que han permitido evaluar y medir de manera cualitativa y cuantitativa su comportamiento desde la ética empresarial, de acuerdo con los cinco valores. Además, se logra reflexionar sobre el significado de la ética empresarial en la formación de los estudiantes de la Maestría en Administración MBA de la Universidad Santo
Tomás Seccional Bucaramanga y cómo trasciende hacia sus organizaciones por medio de sus prácticas profesionales pertinentes para brindar soluciones a necesidades internas y de su entorno.

De igual forma, el estudio identifica aquellas experiencias que demuestran cómo la ética empresarial se ha convertido en una estrategia de cooperación, equidad y productividad sostenible en la que han participado los egresados de la Maestría en Administración.

Además, esta investigación aporta nuevo conocimiento, ya que no existen estudios en la Universidad Santo Tomás Seccional Bucaramanga que indaguen sobre el comportamiento ético de los egresados de la Maestría en Administración MBA, teniendo en cuenta el modelo de formación integral de la Universidad y cómo trasciende hasta sus organizaciones.

Asimismo, los resultados de investigación también ofrecen información a las organizaciones sobre ética empresarial en la gestión de los procesos de servicio al cliente, que les permite conocer y plantear nuevas estrategias para satisfacer de la mejor manera las necesidades de sus usuarios y consumidores.

La ética empresarial es un tema de especial interés para las organizaciones en el mundo, como lo es para la Unesco a través de su programa en pro de la ciencia: Marco General de Acción (Unesco, 1999). Adicional a esto, cada vez existen más personas que, como consumidores o usuarios de productos y servicios, están interesadas en denunciar prácticas que atentan contra sus derechos y por consiguiente son el resultado de un problema de ética en las empresas. 
Dentro del análisis de los resultados se concluye que los objetivos de la investigación se cumplieron gracias a la información obtenida a través del cuestionario aplicado a los egresados de la Maestría en Administración. En las respuestas se aprecian de manera clara cuáles son sus percepciones y las actitudes como líderes de los procesos de servicio al cliente, de acuerdo con los valores planteados por el filósofo Horacio Martínez Herrera como respeto, honestidad, justicia, solidaridad y responsabilidad.

Figura 1. Percepción de respeto con los clientes y consumidores

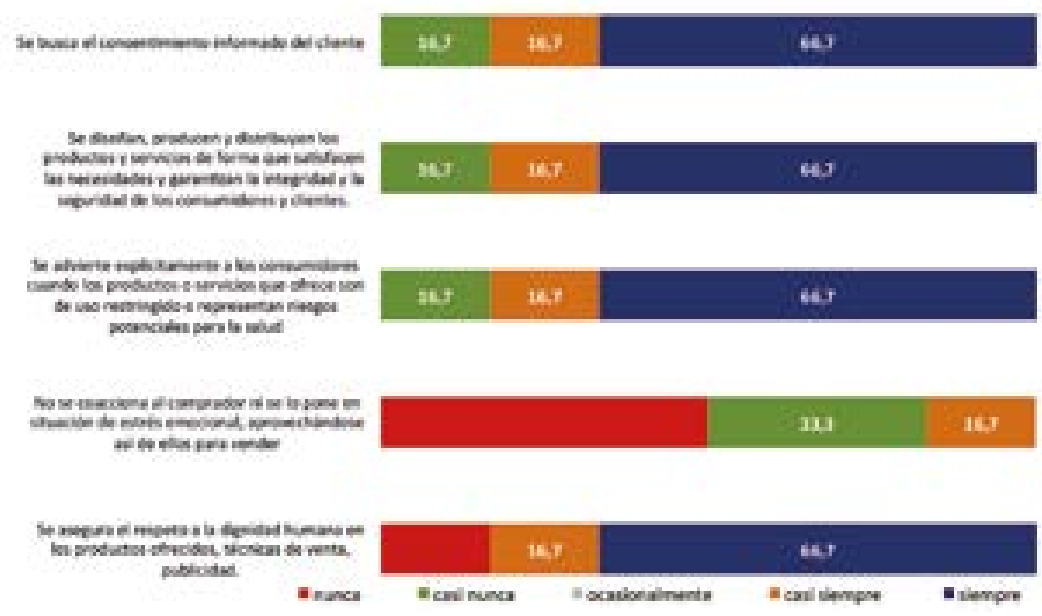

Fuente: elaboración propia.

Frente al concepto de respeto se observa que más del $50 \%$ de los egresados afirman que la información que la empresa brinda a sus clientes utiliza técnicas de venta y publicidad adecuada que advierte de manera clara sobre la forma de producción, distribución, restricciones y riesgos potenciales de los productos y servicios. Sin embargo, es importante mencionar que también se debe poner atención al otro $50 \%$ de las respuestas en las que se manifiesta que no siempre se mantiene una actitud de respeto con el consumidor (figura 1)

Figura 2. Percepción de solidaridad con los clientes y consumidores

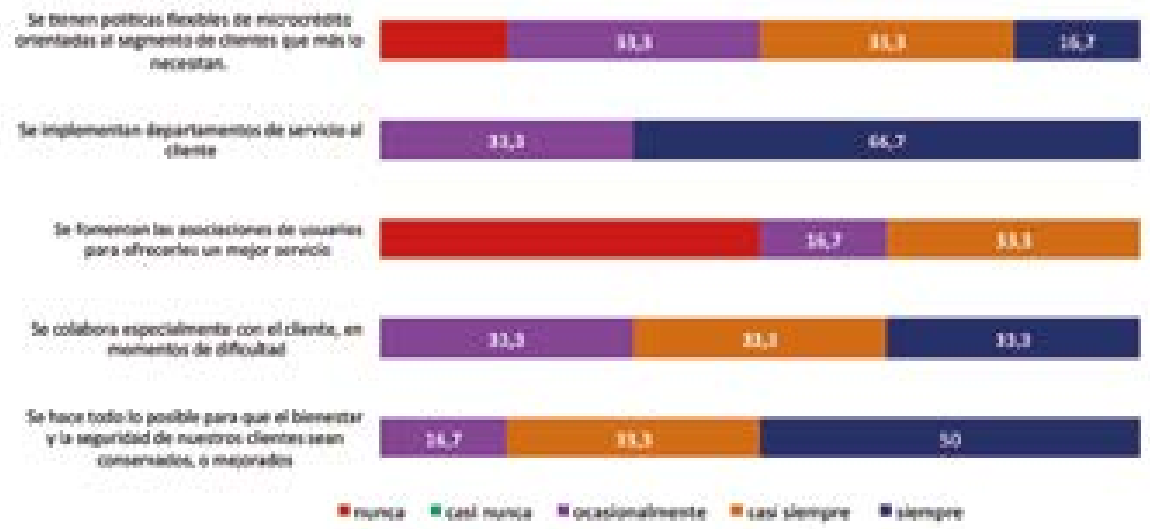


En cuanto a la solidaridad con el cliente, las respuestas de los egresados en la encuesta indican que algunas de sus empresas se preocupan por el bienestar y la seguridad de los clientes y cuentan con un departamento de Servicio al Cliente. Pero igual a la pregunta sobre respeto en las respuestas de este valor también se encuentra que apenas el $50 \%$ actúa de manera favorable en la atención al consumidor, la otra mitad carece de medios que fomenten la solidaridad, incluso el fomento a la asociación de usuarios es poco (figura 2).

Figura 3. Percepción de justicia con los clientes y consumidores

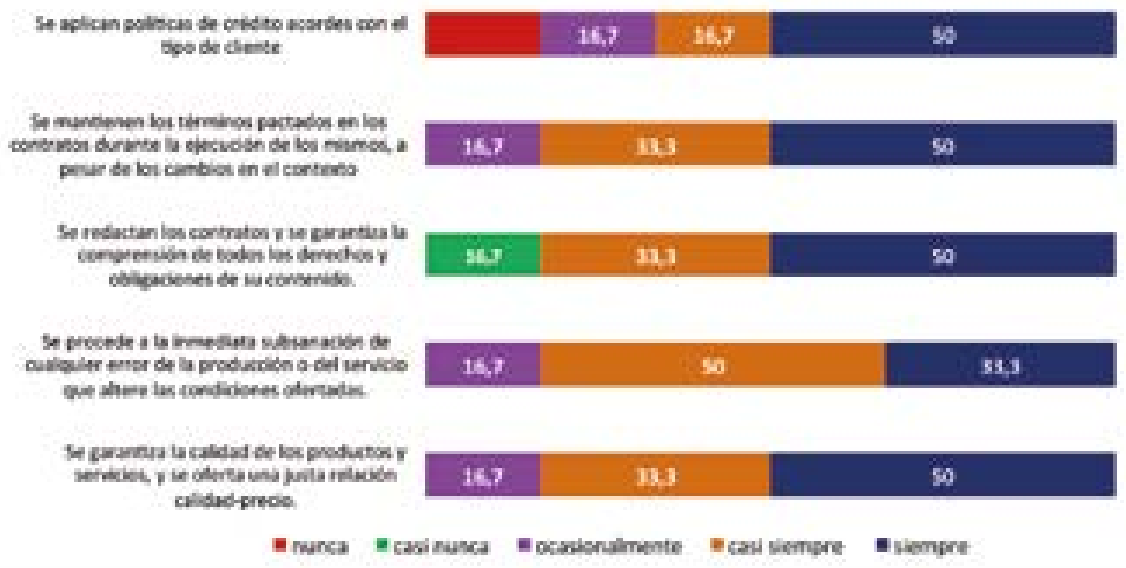

Fuente: elaboración propia.

Por otra parte, la justicia es otro de los valores que se cuestiona en cuanto a su cumplimiento, porque bien es cierto existe una percepción favorable en las respuestas que brindaron los egresados a través del cuestionario, también aparece información que preocupa porque no siempre en todas las organizaciones se garantiza un equilibrio entre el precio y el estado de los productos, ni se mantienen los términos de los contratos pactados con sus usuarios, y peor aún, en el servicio posventa el $66.7 \%$ de las empresas solo procede algunas veces de manera inmediata a subsanar los errores (figura 3 ).

Figura 4. Percepción de responsabilidad con los clientes y consumidores

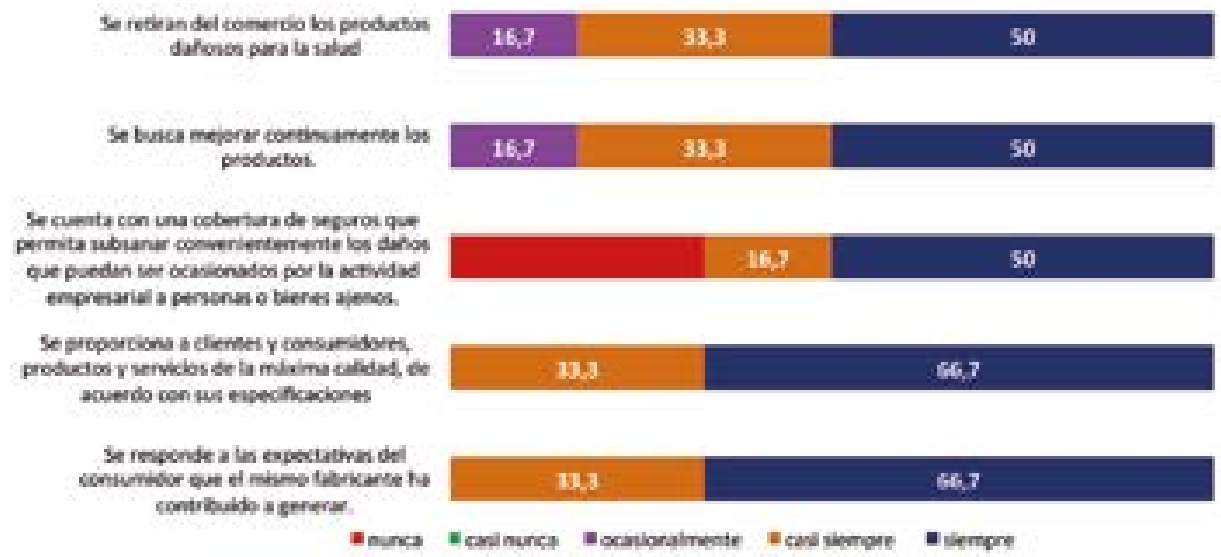


En el tema de la responsabilidad se destaca que en el $66.7 \%$ de las respuestas de los egresados de la Maestría en Administración es positiva, ya que sus empresas responden a las expectativas de sus consumidores, proporcionando productos y servicios de máxima calidad, de acuerdo con sus especificaciones. No obstante, es necesario, de acuerdo con el $50 \%$ de otras respuestas, atender otras circunstancias que no se cumplen en su totalidad, como la búsqueda del mejoramiento de los productos y servicios o de medidas que permitan evitar los daños que puedan ocasionar a sus consumidores (figura 4).

Figura 5. Percepción de honestidad con los clientes y consumidores

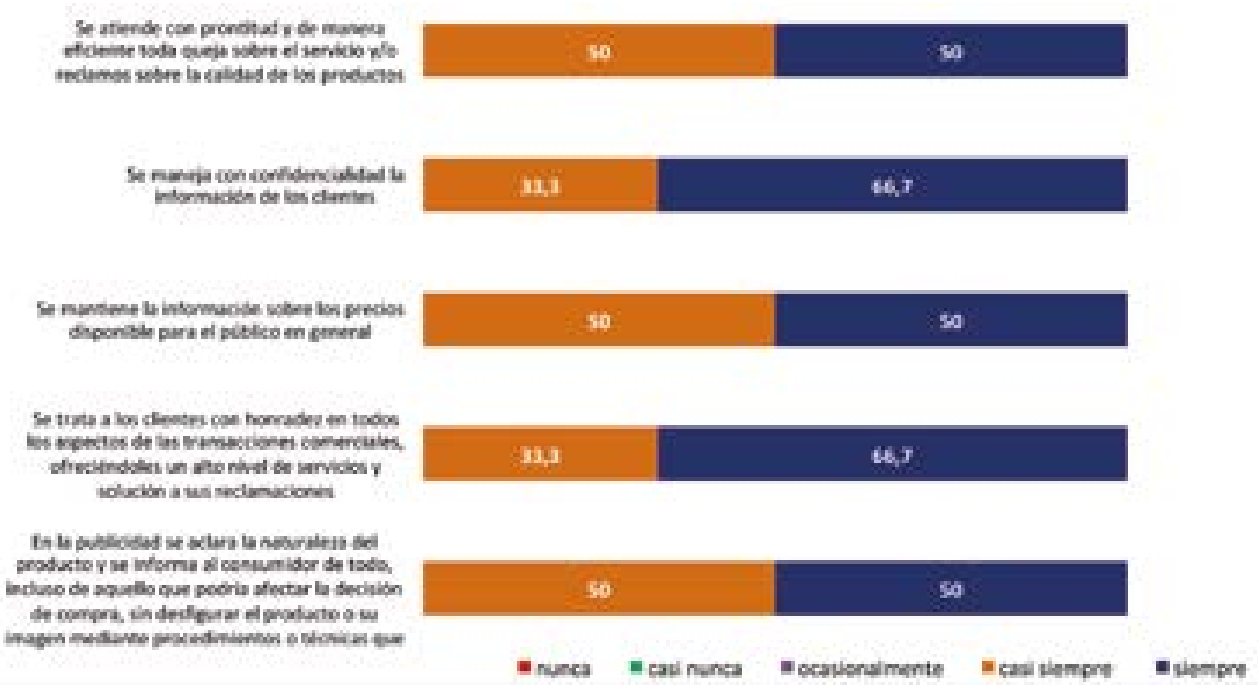

Fuente: elaboración propia.

Finalmente, en cuanto al último valor relacionado a la honestidad se concluye con un $66.7 \%$ de favorabilidad que en todos los procesos de servicio al cliente se conserva la confidencialidad de la base de datos de los clientes, se atienden sus reclamaciones de manera inmediata y se les trata con honradez en todos los aspectos comerciales, como la publicación de precios actualizados, la naturaleza de los productos, entre otros aspectos importantes que afectan la decisión de compra del producto o servicio, mediante procedimientos o técnicas que puedan inducir a engaños o confusiones (figura 5).

\section{Conclusiones}

En general, se puede concluir que existe un porcentaje más alto, el $53.3 \%$ de respuestas que indican que existe una percepción y una actitud favorable sobre ética empresarial, de manera especial en cuanto a valores como justicia y responsabilidad hacia los consumidores por parte de los egresados de la Maestría en Administración de la Universidad Santo Tomás Seccional Bucaramanga, graduados en los años 2012 y 2013, y que actualmente lideran procesos de servicio al cliente en empresas ubicadas en el área metropolitana de Bucaramanga. 
Por otra parte, al analizar las respuestas de los egresados y comparar las variables sociodemográficas se concluye que existe una diferencia significativa entre hombres y mujeres en la actitud hacia la solidaridad y la justicia, siendo las mujeres más solidarias y justas. En cuanto al estrato socioeconómico no existe alguna diferencia, es decir, las percepciones y las actitudes sobre ética son las mismas en los seis niveles. Por último, se aprecia una diferencia que determina que tanto el respeto y la responsabilidad tienen un alto valor para los egresados de acuerdo con los años de servicio en su empresa.

En cuanto al contexto analizado sobre la situación de países como Colombia, donde los índices de corrupción son altos, es importante seguir realizando estudios que permitan analizar las causas intrínsecas que están llevando a los profesionales de las distintas instituciones de educación superior a incurrir en delitos a través de sus organizaciones, afectando de manera significativa el desarrollo económico y social de quienes tienen algún tipo de necesidad y que buscan satisfacerla a través de alguna labor, productos o servicios que ofrece el Estado o las empresas privadas.

Teniendo en cuenta que este es un primer trabajo en el área de la responsabilidad social empresarial y, de manera específica, sobre la percepción ética de los egresados en sus organizaciones, es importante mencionar que si bien se cumplieron los objetivos de la investigación tomando como base una población representativa de 12 egresados que cumplían con el perfil, se recomienda para futuras investigaciones realizar estudios con poblaciones más amplias que permitan generalizar más la información y cruzarla con otro tipo de públicos que se relacionen con la empresa, como trabajadores, clientes, proveedores, accionistas, entre otros.
Por otra parte, también se recomienda aunar unos nuevos estudios, si se requiere, en el trabajo que viene desarrollando la Universidad Santo Tomás sobre la consolidación de una oferta académica en pregrado y posgrado que garantice a sus estudiantes un modelo educativo caracterizado por su formación integral humanista, que le brindará como egresado las condiciones necesarias para que desde el ejercicio reflexivo responda con actitud y compromiso personal y profesional a favor de la vida, cuestionando y rechazando cualquier mecanismo de delito, como la corrupción, que atente contra la construcción democrática de las verdades, la dignificación de la persona humana y de la comunidad.

De igual forma, y teniendo en cuenta la población de estudio de este trabajo, se plantea para nuevas investigaciones la creación e implementación de un espacio académico en el marco de la propuesta curricular de la Maestría en Administración con núcleos problémicos que interpreten el fenómeno de la ética empresarial desde enfoques como el humanista y el social. Este ejercicio se plantea para favorecer el pensamiento propositivo, incluyente y respetuoso de la diversidad y como promotor del desarrollo humano, a partir de los valores institucionales que promueve la Universidad Santo Tomás entre sus estudiantes y egresados, mediante herramientas teóricas y metodológicas que permitan proyectarse desde el ejercicio profesional hacia un compromiso ético con responsabilidad social.

Esta propuesta, además, puede continuar fortaleciendo el enfoque de la formación humana integral desde lo académico en los posgrados de la Universidad Santo Tomás Seccional Bucaramanga, con una ruta formativa que parte del Modelo Educativo Pedagógico Institucional y de las líneas de investigación que dan origen 
a las especializaciones y maestrías que hacen parte de cada una de las divisiones académicas: Ciencias Jurídicas y Políticas, Ingenierías y Arquitectura, Ciencias de la Salud, Ciencias Económicas y Administrativas.

De igual manera se recomienda para unos nuevos estudios continuar con la aprehensión de la ética empresarial dentro de las prácticas profesionales en el ámbito de los negocios y de la relación con clientes y consumidores, esto, por supuesto, aunado a un trabajo de seguimiento establecido mediante mecanismos de evaluación sistemática con los egresados de la Maestría en Administración de la Universidad Santo Tomás, para monitorear su gestión desde los comportamientos éticos que presentan a través de sus cargos en organizaciones donde cumplan funciones estratégicas.

Finalmente, se espera que con este estudio exploratorio se logre dar inicio a otros proyectos que permitan identificar finalmente cuáles son las razones que conducen a los profesionales a utilizar su conocimiento en conductas indebidas, y cómo estos actos se convierten en un mal endémico y estructural que impregna y contagia la mentalidad de otros integrantes que terminan por afectar sus empresas, hasta el punto, incluso, de deteriorar los núcleos familiares, acabar con la economía y desacelerar el desarrollo y la calidad de vida de una sociedad.

\section{Referencias}

Arbaiza, L. (2010). Responsabilidad social: tarea compartida. ESAN: Business.

CEPAL. (2008, enero). Boletín FAL No 257. Responsabilidad social empresarial: ventaja competitiva en las organizaciones. CEPAL.

Consejo Nacional de Educación Superior - CESU. (2013). Acuerdo por lo Superior 2034 Propuesta de política pública para la excelencia de la educación superior en Colombia en el escenario de la paz. Bogotá: Multi-impresos S.A.S.

Morín, E. (2002a). Los siete saberes necesarios para la educación del futuro. Buenos Aires.

Sampieri, R. H., Fernández Collado, C., \& Baptista Lucio, M. d. (2010). Metodología de la Investigación. México: McGraw-Hill.

Transparencia Internacional. (2015). Corruption Perceptions Index. Alemania.

Universidad Santo Tomás. (2004). Proyecto Educativo Institucional - PEI. Bogotá D.C.: USTA.

Universidad Santo Tomás. (2014). Recuperado el 14 de septiembre de 2014, de www.ustabuca.edu.co: http://www.ustabuca.edu.co/gpresenzia/vista/tpl/ ustabmanga/maestria-en-administracion.html

Universidad Santo Tomás, Departamento Humanidades. (2014). Las humanidades como instancia de la formación integral en la Universidad Santo Tomás. Bogotá: USTA Ediciones. 